\title{
Surgical treatment of carcinoma of the oesophagus
}

\author{
W. P. Cleland \\ F.R.C.P., F.R.C.S., F.A.C.S.
}

\section{Hammersmith Hospital and Royal Postgraduate Medical School, London}

\begin{abstract}
Summary
There is a place for a radical surgical approach to the problem of carcinoma of the oesophagus and it should be our first aim to determine whether such an approach is justified. If radical treatment can not be carried out then the surgeon's only concern is with the preservation of the function of swallowing.
\end{abstract}

RADICAL treatment of carcinoma of the oesophagus enjoys an indifferent reputation but reports from reputable centres where the operation is done on a regular basis suggest that this gloomy picture is not justified. McKeown (1972) in his Hunterian Lecture, showed that the 3-year survival rate for growths at the gastro-oesophageal junction was $21.6 \%$. For growths in the lower third and mid third of the oesophagus the situation was better with a 3 -year survival of $36.4 \%$ and $35.6 \%$ respectively. Collis (1972) similarly reports a survival rate of $30 \%$ at 2 years, $15 \%$ at 5 years and $13 \%$ at 10 years. In each case the operative mortality was in the region of $10-15 \%$. These figures can be reproduced from many of the best centres in this country and abroad and should encourage a radical outlook in suitable individuals.

\section{Scope of surgical treatment}

The prime aim of surgical treatment should thus be radical resection of the tumour and all its known gland distribution areas combined with reconstitution of the digestive tract. Such an approach can only be carried out after all investigations have failed to provide evidence of inoperability. Should the condition already be inoperable the surgeon's duty then is one of providing a suitable channel for the passage of food and drink. This can often be very effectively provided by the use of an indwelling tube and of these the new Celestin tube has much to offer in that the tube is armour plated to prevent kinking and has a radio-opaque strip for radiological identification. Palliation of this sort should be instituted once the patient's nutrition begins to suffer. There is an intermediate group of patients who have been considered suitable for exploration but found to be inoperable at laparotomy or thoracotomy. In such cases one might well use the exposure to insert an indwelling tube or one may, with justification, consider removing the primary and restore continuity of the alimentary canal by simple means. Such a procedure would have little effect upon the length of survival but would almost certainly enable the patient to swallow without discomfort until secondary deposits had their way.

\section{Assessment for radical surgery}

Clearly, if a radical policy is to be pursued assessment of suitability for surgery is of paramount importance. This should consist of the following.

(1) Oesophageal inspection. The most important aspects of oesophagoscopic inspection is the determination of mobility of the oesophagus and fixity of the growth. A fixed, rigid oesophageal tumour would suggest infiltration into the mediastinum.

(2) Bronchoscopic examination should be carried out in every case and careful attention paid to the region of the carina and the right and left main bronchi for any evidence of distortion or invasion. Fixity of the bronchial tree by a mid thoracic growth is a sinister sign.

(3) Tomographic examination of the mediastinum in the region of the bifurcation of the trachea is of particular value in mid oseophageal growths and may provide evidence about the extent of the lesion and whether vital structures are being invaded.

(4) Liver scan. In view of the frequent metastatic spread to the liver, scanning would seem to be advisable although interpretation of results is still somewhat uncertain.

Finally some attention must be paid to the general and nutritional state of the patient although it is surprising how often a thin and wasted individual will prove to have a removable tumour.

\section{Preparation for surgery}

In addition to the routine preparation for surgery particular attention should be paid to the nutrition of the patient as his intake of calories and vitamins has often been depleted for some weeks or months. As long a period as possible should be spent in the building up process using Complan as a basis for 
those who cannot take solids. Particular attention ought to be paid to the condition of the mouth and any signs of dental infection dealt with radically. A period of time spent with the physiotherapist may save considerable disappointments in the postoperative period. It is wise in every case to pay some attention to the preparation of the bowel, whatever method of reconstruction is contemplated. Our routine is as follows. Neomycin G. 1, Bacitracin 100,000 units, Nystatin 250,000 units made up to $30 \mathrm{ml}$ with water. This may be given 4 times a day for $48 \mathrm{hr}$ pre-operatively or, if less time is available, can be given hourly for $4 \mathrm{hr}$ and then 4 hourly for $12 \mathrm{hr}$.

\section{Surgical technical problems}

I do not want to deal in any detail with the surgical problems involved but merely would like to discuss some of the principles.

Approaches. For all growths at the gastrooesophageal junction a thoraco-abdominal incision is ideal but as far as possible every attempt should be made to preserve the function of the diaphragm as good lung function subsequently is largely dependent upon its efficiency.

For growths in the middle third of the oesophagus most surgeons agree that a right-sided approach is preferable for radical surgery. Only from the right side is one able to dissect oesophageal tumours from the aorta, the bronchus, the trachea and the pericardium. In such cases an abdominal approach is required initially for mobilizing the stomach and this is subsequently followed by a separate thoracotomy. The anastomosis between stomach and oesophagus can well be carried out in the chest although some prefer a cervical approach with the performance of the anastomosis at this level.

As regards the excision itself the tumour extends for only a limited distance upwards though it may extend downwards more considerably. A clearance of $4-5 \mathrm{~cm}$ above the upper limits of the visible and palpable tumour is usually regarded as sufficient clearance but the whole of the oesophagus below the growth as far as the cardia or even beyond should be excised. Equally important is the excision of the gland bearing areas. In the thorax the lymph nodes are all in the paraoesophageal region and these would normally be excised at the time of the oesophageal mobilization but the dissection should be carried as high up as possible and include the paratracheal nodes on the right side. It is not, of course, practicable to remove the upper paratracheal nodes on the left side.

\section{Restoration of continuity}

This is, to a large extent, a personal decision and I do not propose to deal with it at any great length. For growths at the gastro-oesophageal junction it is clearly advisable to remove the whole of the stomach and one must therefore use either jejunum or colon for restoration. For mid oesophageal growths it is usually unnecessary to remove the stomach and this can be used for an intra-thoracic anastomosis and with it anastomosis can be readily effected as high up as the thoracic inlet. Oesophago-gastrostomy has been criticized in the past because of complications of reflux oesophagitis and peptic ulceration. These complications seem less likely if the anastomosis is done fairly high up in the thorax.

The other major complications after oesophagec tomy are anaemia due to the loss of the stomach and all patients should be maintained on regular injections of vitamin $\mathrm{B}_{12}$; diarrhoea or steatorrhoea are not uncommon and probably related to vagal disturbances. Nutrition is often somewhat of a problem but the patient should be advised to indulge in frequent, small meals six or more a day rather than the more orthodox pattern.

\section{References}

Collis, J.L. (1972) The long-term clinical state after resection with gastro-oesophagestomy. Surgery of the Oesophagus (Ed. by R. Abbey-Smith and R. E. Smith.) London: Butterworths.

McKeown, R.C. (1972) Trends in oesophageal resection for carcinoma. Annals of the Royal College of Surgeons of England, 51, 213. 